\title{
Field trial of primary and booster dose of inactivated vaccine against bovine respiratory bacteria in young Holstein calves
}

\author{
Kazusa Mori ${ }^{1}$, Toshihide Kato ${ }^{1}$, Osamu Yokota ${ }^{2}$, Hiromichi Ohtsuka $^{1 凶}$ \\ ${ }^{1}$ Rakuno Gakuen University Animal Medical Center, Ebetsu, Hokkaido 069-8501, Japan \\ ${ }^{2}$ F. SIDE Veterinary Service, Sapporo, Hokkaido 004-0072, Japan \\ ohtsuka@rakuno.ac.jp
}

Received: September 26, 2019 Accepted: June 1, 2020

\begin{abstract}
Introduction: The objective of this research was to evaluate the antibody response to multiple doses of an inactivated mixed vaccine against Histophilus somni, Pasteurella multocida, and Mannheimia haemolytica, and to investigate the influence of age at time of vaccination in the field. Material and Methods: Healthy female Holstein calves received the vaccine at the age of 5-12 days and 2,3, or 4 weeks later in the first experiment or at 1,2, or 3 weeks of age and 4 weeks later in the second. Blood samples were collected at each vaccination and 3 weeks after the booster dose. Based on the antibody titres after the vaccinations, calves were divided into positive and negative groups for each of the bacteria. Calves in the control group were vaccinated only once at the age of 19-26 days. Results: Antibody titres against H. somni and P. multocida were significantly increased by the booster. After the second vaccinations, the titres against each bacterium were higher than those of the control group, and the M. haemolyticapositive percentage in calves with high maternal antibody levels (MAL) exceeded that in calves with low MAL. In the first experiment, a majority of the $M$. haemolytica-positive calves tended to have received the primary dose at seven days of age or older. Conclusion: A booster dose of the inactivated bacterial vaccine in young Holstein calves increased antibody production and overcame the maternal antibodies. Calves should be vaccinated first at seven days of age or older.
\end{abstract}

Keywords: bovine respiratory disease, young calves, early vaccination.

\section{Introduction}

Bovine respiratory disease (BRD) is one of the main causes of death in pre-weaned calves and weaned dairy heifers (20). Histophilus somni, Pasteurella multocida, and Mannheimia haemolytica are frequently implicated as the bacterial pathogens (5), and BRD is usually treated with antimicrobial drugs. However, the antimicrobial resistance of BRD pathogens has increased in recent years (3). In addition, the prognosis of BRD is poor. Dairy heifers which had been treated for pneumonia during the first three months of life showed 2.5-times higher mortality after 90 days of age than heifers which had not been treated (21). Thus, prevention of BRD is very important in order to reduce economic losses.

Young calves have a naive immune system (10). The bovine neonate is born agammaglobulinaemic and relies on colostrum for the establishment of passive immunity (7). For this reason, protection provided by innate immune mechanisms and by transferred maternal antibodies plays an important role in the calf's defence against infectious diseases (10). However, in Holstein calves the transferred maternal antibody titre against $M$. haemolytica decreases to a low level at 5 weeks of age and seroconversion occurs via the calf's own immune response (9). Therefore, young calves should be vaccinated at an early age to prevent BRD.

Early vaccination of young calves is nevertheless far from universal. A number of studies have demonstrated that acquired antibodies may compromise the effect of vaccination $(7,14,16)$. It has been reported that Holstein dairy calves which were vaccinated at two and four weeks of life with live M. haemolytica vaccine and calves which were vaccinated at one month of age with inactivated $M$. haemolytica vaccine did not show increases in antibody titres within one month after the primary vaccination $(9,11)$.

There are live and inactivated vaccines available in the marketplace which protect against BRD pathogens 
and those vaccines may help to stimulate fast and steady antibody production $(14,18)$. At the same time, both types of vaccine have disadvantages. Live attenuated vaccines may cause the illness they are designed to prevent. Inactivated vaccines require several administrations because the microbes are unable to multiply in the host and one dose is not sufficient to stimulate the adaptive immune system (2). However, not enough information is available regarding multiple doses of inactivated bovine respiratory bacterial vaccines given to young Holstein calves.

Here, we report antibody responses to a booster dose of inactivated $H$. somni, $P$. multocida, and $M$. haemolytica vaccines given to young Holstein calves in the field. The main objective of this study was to determine whether the vaccine would overcome maternal immunity, and determine the age when calves should be vaccinated.

\section{Material and Methods}

Healthy female Holstein calves were used in this study. All calves were fed adequate amounts of colostrum replacer (Zenoaq, Fukushima, Japan) and received $1 \mathrm{~mL}$ of a commercial vaccine intramuscularly on two different dates. The vaccine used contained inactivated antigens of $H$. somni, P. multocida, and $M$. haemolytica (No. 26-1 strain; Kyoto Biken Laboratories, Inc., Uji, Japan).
In the first experiment, 71 calves were used, of which 56 received the primary vaccination between 5 and 12 days of age. Then these calves were divided into three groups that differed in the time interval to the booster vaccination after the primary dose. Table 1 shows the time schedules of vaccination and blood sampling as well as the sample size of each group. Each calf of the remaining 15 which comprised the control group was vaccinated once between 19 and 26 days of age and no booster vaccinations were administered. In the second experiment, 31 calves were used. The calves were divided into three groups receiving the primary dose at different ages indicated as follows: at one (7-12 days), two (14-19 days), or three weeks of age (21-27 days). Each of the calves received the booster vaccination four weeks after their primary vaccination was administered.

In both experiments, blood was drawn immediately prior to each vaccination, three weeks after the booster dose was administered, and on the time schedules shown in Table 1. Blood samples were collected into plain vacutainer tubes and centrifuged at 3,500 rpm for eight min, and then blood serum was isolated. Serum was kept at $-30^{\circ} \mathrm{C}$ until analysis and serum antibodies against $H$. somni, P. multocida, and M. haemolytica were detected using ELISA as described in a previous study (15). For P. multocida and M. haemolytica, when the antibody titre was detected with less than 100-fold dilution, it was calculated as a 50-fold dilution.

Table 1. Time schedules of vaccination and blood sampling

First experiment

\begin{tabular}{|c|c|c|c|c|c|c|c|c|c|}
\hline $\begin{array}{l}\text { Weeks after primary vaccination } \\
\text { (or first blood sampling) }\end{array}$ & 0 & 1 & 2 & 3 & 4 & 5 & 6 & 7 & Sample size \\
\hline+2 group & Primary* & & Booster & & & BS & & & $\mathrm{n}=21$ \\
\hline+3 group & Primary* & & & Booster & & & $\mathrm{BS}$ & & $\mathrm{n}=16$ \\
\hline+4 group & Primary* & & & & Booster & & & $\mathrm{BS}$ & $\mathrm{n}=19$ \\
\hline Control group & $\mathrm{BS} * *$ & & Vaccination & & & & $\mathrm{BS}$ & & $\mathrm{n}=15$ \\
\hline
\end{tabular}

*Experimental calves received primary vaccination at the age of 5-12 days

**In control group calves, blood was drawn at this period and calves received vaccination only once 2 weeks later

\begin{tabular}{|c|c|c|c|c|c|c|c|c|}
\hline & Primary vaccina & & Booster vac & nation & Blood san & ling & Sample size & \\
\hline $7-12$ day group & age of $7-12 \mathrm{da}$ & & \multirow{2}{*}{\multicolumn{2}{|c|}{$\begin{array}{l}2,3 \text { or } 4 \text { weeks after } \\
\text { primary vaccination }\end{array}$}} & \multirow{2}{*}{\multicolumn{2}{|c|}{$\begin{array}{c}3 \text { weeks after booster } \\
\text { vaccination }\end{array}$}} & $\mathrm{n}=40$ & \\
\hline 5-6 day group & age of 5 or $6 \mathrm{da}$ & & & & & & $\mathrm{n}=16$ & \\
\hline & Maternal antibody level & Pri & vaccination & Boc & accination & & d sampling & Sample size \\
\hline Mh High group & Mh: 200 or over & \multirow{4}{*}{\multicolumn{2}{|c|}{ the age of $7-12$ days }} & \multirow{4}{*}{\multicolumn{2}{|c|}{$\begin{array}{l}\text { 2, } 3 \text { or } 4 \text { weeks } \\
\text { after primary vaccination }\end{array}$}} & \multirow{4}{*}{\multicolumn{2}{|c|}{$\begin{array}{c}3 \text { weeks } \\
\text { after booster vaccination }\end{array}$}} & $\mathrm{n}=21$ \\
\hline Mh Low group & Mh: under 200 & & & & & & & $\mathrm{n}=19$ \\
\hline Pm High group & Pm: 100 or over & & & & & & & $\mathrm{n}=25$ \\
\hline Pm Low group & Pm: under 100 & & & & & & & $\mathrm{n}=15$ \\
\hline
\end{tabular}

Second experiment

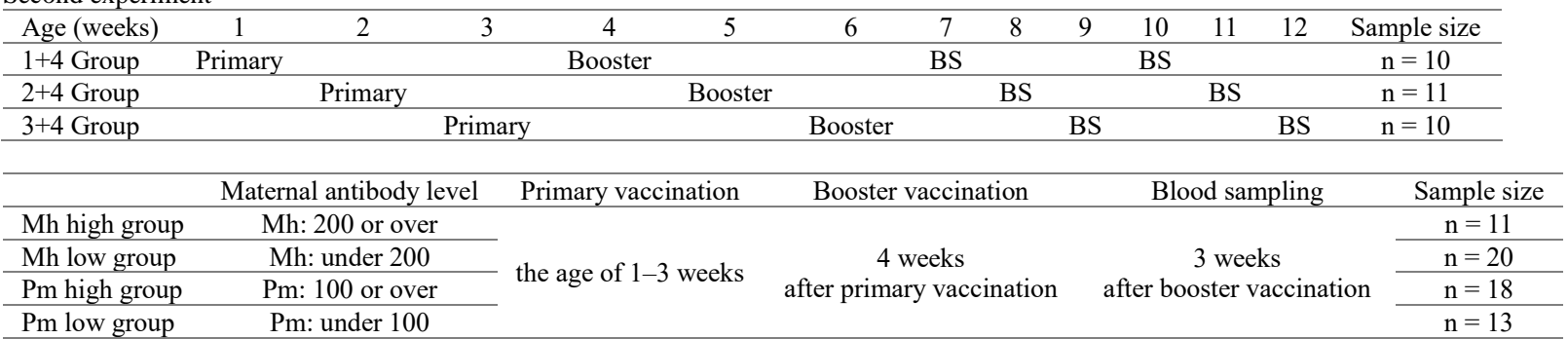

Primary - primary vaccination; booster - booster vaccination; BS - blood sampling; maternal antibody level - the titre at primary vaccination; $\mathrm{Mh}-$ M. haemolytica; $\mathrm{Pm}-P$. multocida 
Antibody titres were compared among groups in each experiment at three weeks after the booster dose as well as among sampling points within the same group. Taking the paper of Otomaru et al. as a guide (17), titres more than 0.604 (H. somni), 100 (P. multocida), and 200 $(M$. haemolytica) were considered antibody positive. Based on the titres at three weeks after the booster dose, calves were divided into positive and negative groups for H. somni, P. multocida, or M. haemolytica. Age in days at the primary vaccination was compared between the M. haemolytica-positive and -negative calves, or the $H$. somni-positive and negative calves in the first experiment.

In the first experiment, titres at three weeks after the booster dose were also compared between calves which received the primary vaccination at the age of $7-12$ days (7-12 day group) and at the age of 5 or 6 days (5-6 day group) in all three groups. The calves which received the primary vaccination at 7-12 days of age in the first experiment and all of those in the second experiment were divided further by the maternal antibody levels (MAL) at the time of the primary dose into a $M$. haemolytica high group (200 and over) and low group (under 200) or a P. multocida high group (100 and over) and low group (under 100). The titres after vaccinations were compared between the M. haemolytica high and the low groups and between the P. multocida high and low groups in each experiment (Fig. 4).

Data were expressed as geometric mean \pm SE. All analyses were performed using XLSTAT 2018.7 (Addinsoft, Paris, France). The differences among groups were examined using the Kruskal-Wallis, SteelDwass-Critchlow-Fligner, Kolmogorov-Smirnov, and chi-squared tests. The differences among sampling points were examined using the Friedman test. $P$ values less than 0.05 were considered statistically significant.

\section{Results}

Figure 1 presents titre change of antibodies to each bacterium over time for the groups by their interval between primary and booster vaccination. Antibody titres against $H$. somni, P. multocida, and $M$. haemolytica did not increase with the primary vaccination at three weeks of age or under. In the first experiment, the titres against $H$. somni and $P$. multocida nevertheless significantly increased with the booster vaccination in all groups during the following three weeks $(\mathrm{P}<0.01)$. Although the titres against $M$. haemolytica did not significantly increase with the booster vaccination, the titres against every bacterium after the booster dose were significantly higher than those of the control group $(\mathrm{P}<0.01)$.

In the first experiment, more than $90 \%$ of calves on average were $H$. somni or $P$. multocida positive at three weeks after the booster vaccination. However, also on average, more than $40 \%$ of calves were M. haemolytica negative and almost no differences in the titres were observed among the $+2,+3$, and +4 groups after the booster vaccination. Table 2 shows the number and percentages of calves determined positive or negative for H. somni, P. multocida, or M. haemolytica at three weeks after the booster dose. There were only slight differences in the percentages of seropositive or seronegative calves for each of the three bacteria among groups in the first experiment. However, the M. haemolytica positive percentage in the 7-12 day group was significantly higher than that in the 5-6 day group, as was this percentage in the $M$. haemolytica high group set against that in the $M$. haemolytica low group in the first experiment, as Table 2 demonstrates.

In the second experiment, antibody production against $H$. somni $(\mathrm{P}<0.01)$ and $P$. multocida $(\mathrm{P}<0.05)$ increased significantly within the three weeks after each booster vaccination. More than $90 \%$ of calves on average were $H$. somni positive and all calves were $P$. multocida positive at three weeks after the booster dose in the second experiment. The titres against M. haemolytica did not increase significantly after either the primary or booster vaccinations, and, also on average, more than $70 \%$ of calves stayed negative for $M$. haemolytica at three weeks after the booster dose. Throughout the second experiment, the differences among the three experimental groups were minor (Fig. 1).

The age at the primary vaccination of the $M$. haemolytica-positive calves in the first experiment was significantly higher than that of the M. haemolyticanegative calves in the +2 group and in the same comparison when all three groups were aggregated $(\mathrm{P}<0.01)$. Although there was no difference in the +3 group, the positive calves were also older than the negative calves in the +4 group $(\mathrm{P}=0.11)$. The proportions of $M$. haemolytica-negative calves which received the primary vaccination aged under seven days were $70.0 \%$ (+2 group), $33.3 \%$ (+3 group), $42.9 \%$ (+4 group) and $52.2 \%$ (all three groups in the first experiment), and the proportions of $M$. haemolyticapositive calves which received it aged at or over seven days were $90.9 \%$ (+2 group), $70.0 \%$ (+3 group), $100 \%$ (+4 group) and $87.9 \%$ (all three groups). Nevertheless, between the $H$. somni-positive and negative calves there was no statistical difference found in the age at the primary vaccination (Fig. 2).

After both vaccinations, the titre against M. haemolytica of the calves in the 7-12 day group was significantly higher than the titres found in the 5-6 day group and the control group $(\mathrm{P}<0.01)$, as Fig. 3 shows. The titre in the 5-6 day group was also higher than that in the control group $(\mathrm{P}=0.073)$ for this bacterium. There were no differences in the titres against $H$. somni and $P$. multocida after vaccinations between the experimental groups (Fig. 3). Calves in the 7-12 day group exhibited increased antibody production against M. haemolytica $(\mathrm{P}=0.051)$, and antibody production against $H$. somni and $P$. multocida increased significantly in both experimental groups $(\mathrm{P}<0.01)$. 
(1)
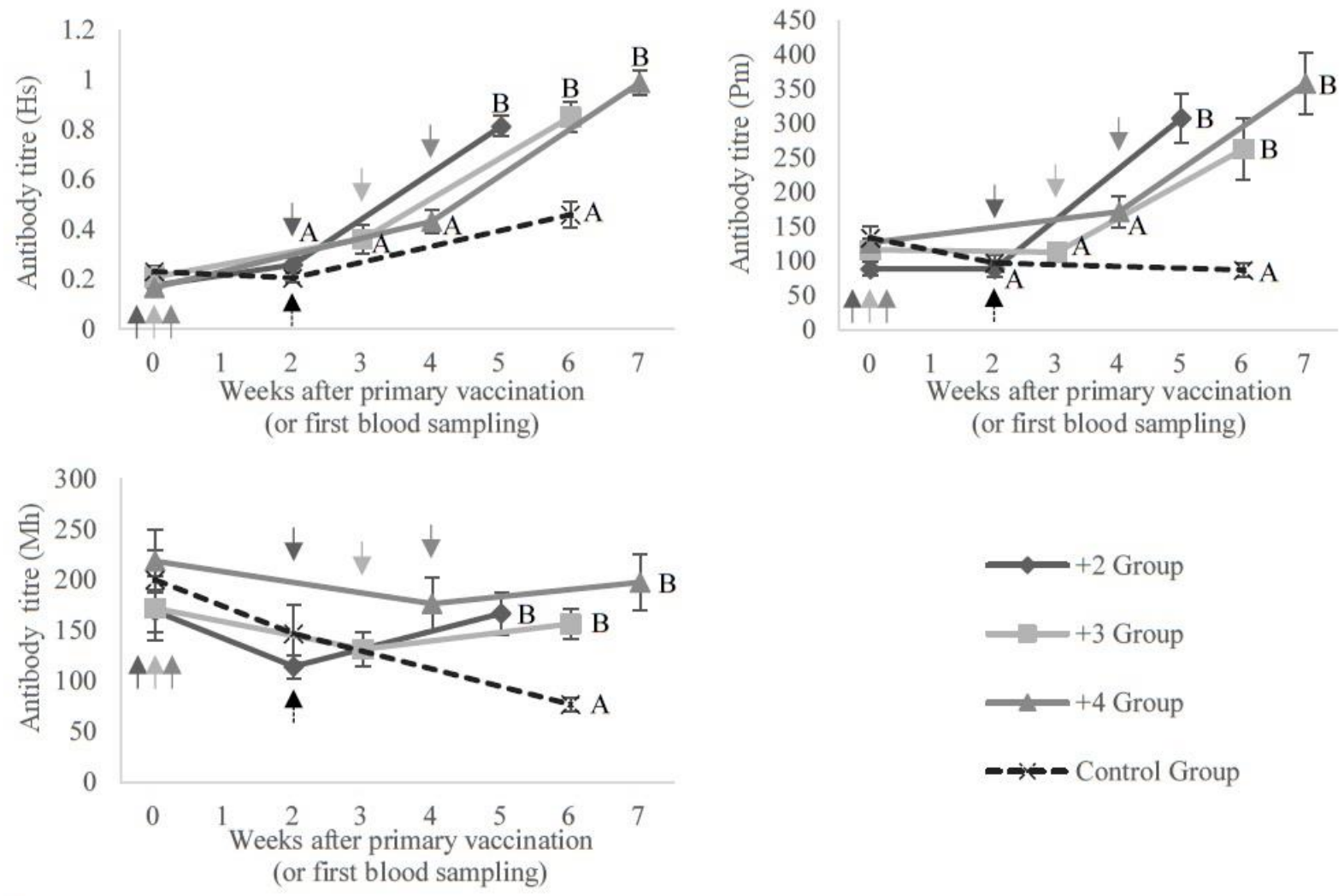

(2)
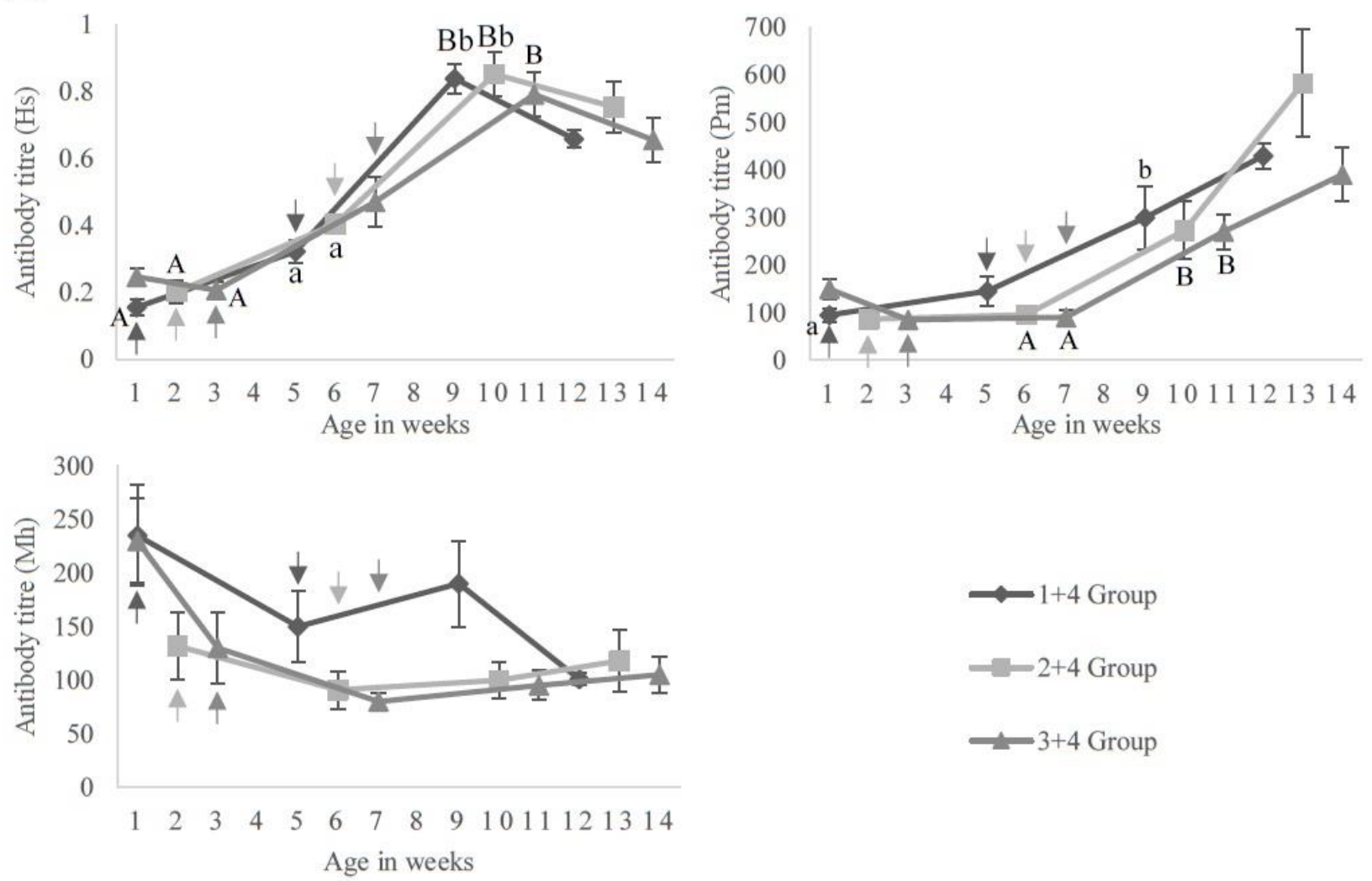

Fig. 1. Antibody response to the vaccinations

Antibody titres against H. somni (Hs), P. multocida (Pm), and M. haemolytica $(\mathrm{Mh})$ in (1) the first experiment and (2) the second experiment Calves received (1) the primary vaccination at the age of 5-12 days and the booster dose at 2, 3 or 4 weeks later or (2) the primary vaccination at 1,2 or 3 weeks of age and the booster dose at 4 weeks later. (1) In control group calves, blood was drawn at the age of 5-12 days and calves received the vaccination only once 2 weeks later (between 19 and 26 days of age). $\mathrm{A}, \mathrm{B}-\mathrm{P}<0.01 ; \mathrm{a}, \mathrm{b}-\mathrm{P}<0.05$; arrows - time of vaccination 
Table 2. Number and percentage of seropositive or seronegative calves at 3 weeks after the booster dose

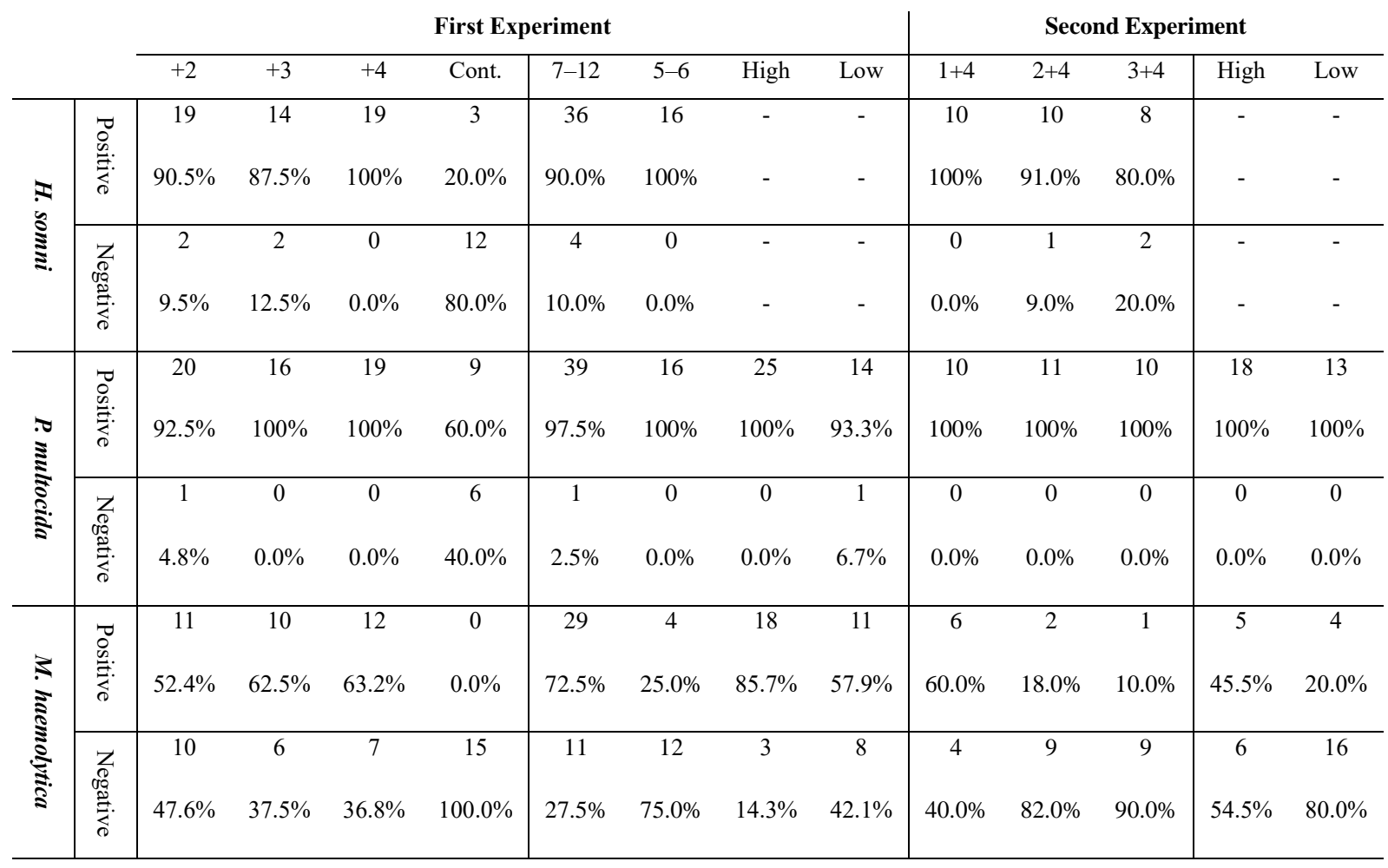

Titres more than 0.604 for $H$. somni, 100 for $P$. multocida, or 200 for $M$. haemolytica were considered antibody positive. 7-12: 7-12 day group; 5-6: 5-6 day group; High: P. multocida or M. haemolytica high maternal antibody group; Low: P. multocida or M. haemolytica low maternal antibody group

(a) Cumulative distributions $(+2 \text { Group })^{* *}$

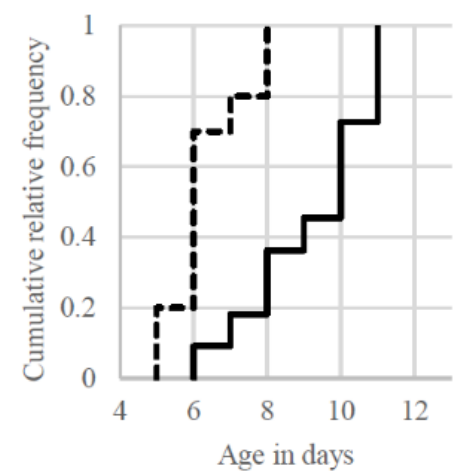

(b)

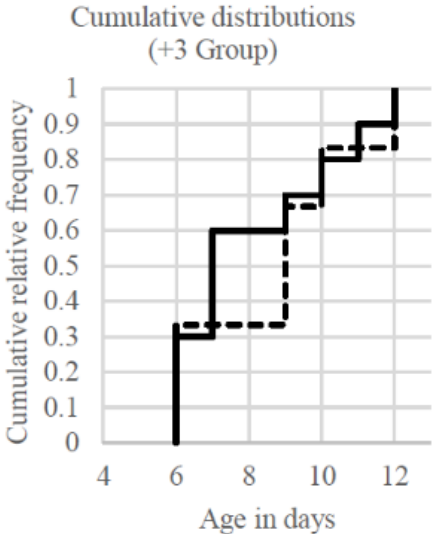

(c) Cumulative distributions $(+4 \text { Group })^{\#}$

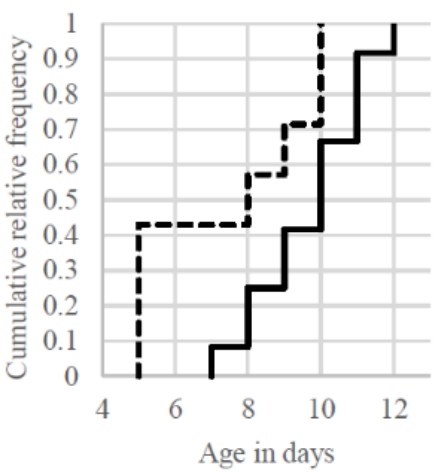

Mh positive

(d)

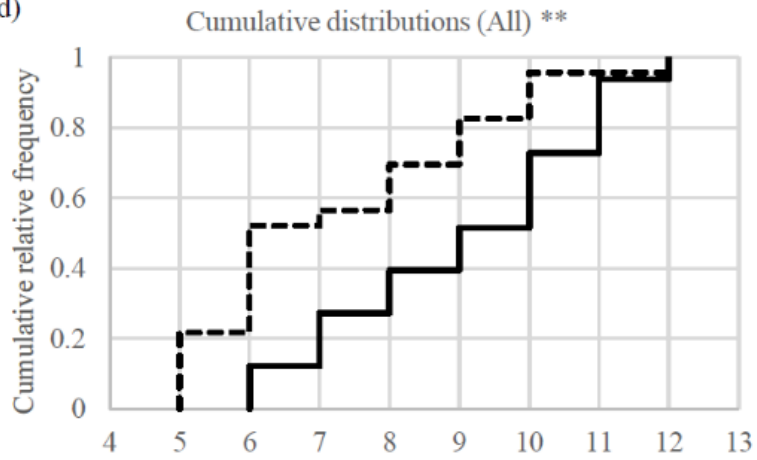

Age in days at primary vaccination

Mh positive (e)

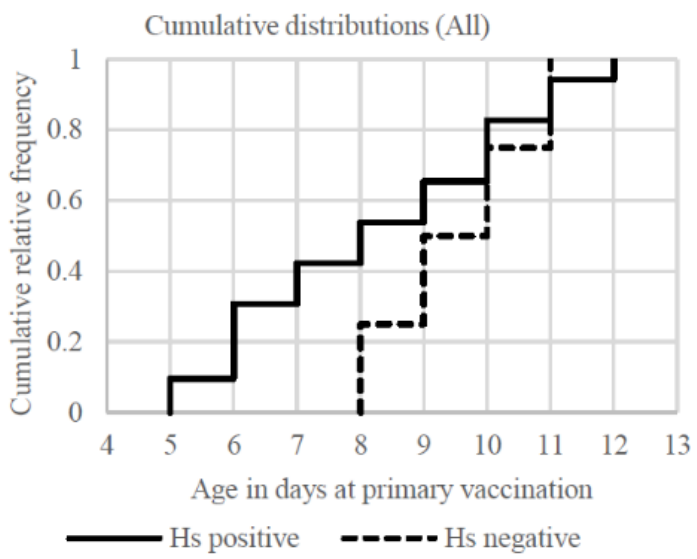

Fig. 2. Age at the primary vaccination

Cumulative distribution of age in days at the time of the primary vaccination in the first experiment; a-d - comparison between $M$. haemolytica (Mh) positive and negative; $\mathrm{e}-$ comparison between $H$. somni $(\mathrm{Hs})$ positive and negative; $* *-\mathrm{P}<0.01 ; \#-\mathrm{P}=0.11$ 

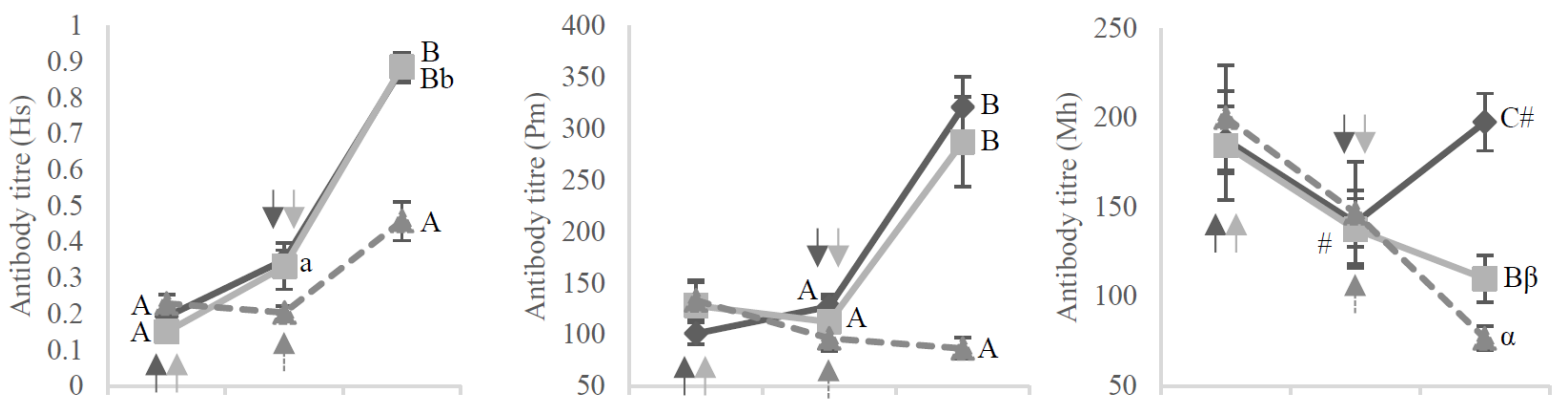

$\longrightarrow$ 7-12 Days Group

5-6 Days Group

$-4-$ Control Group

Fig. 3. Antibody response to the vaccination among calves of different ages at the primary dose

Antibody titres against $H$. somni $(\mathrm{Hs}), P$. multocida $(\mathrm{Pm})$, and $M$. haemolytica $(\mathrm{Mh})$ of calves which received the primary vaccination at the age of 7-12 days (7-12 day group) and 5 or 6 days (5-6 day group). Calves were also vaccinated 2, 3, or 4 weeks later. A, B, C - P < 0.01 ; $\mathrm{a}, \mathrm{b}-\mathrm{P}<0.05 ; \#-\mathrm{P}=0.051 ; \alpha, \beta-0.073$; arrows - time of vaccination

(1)
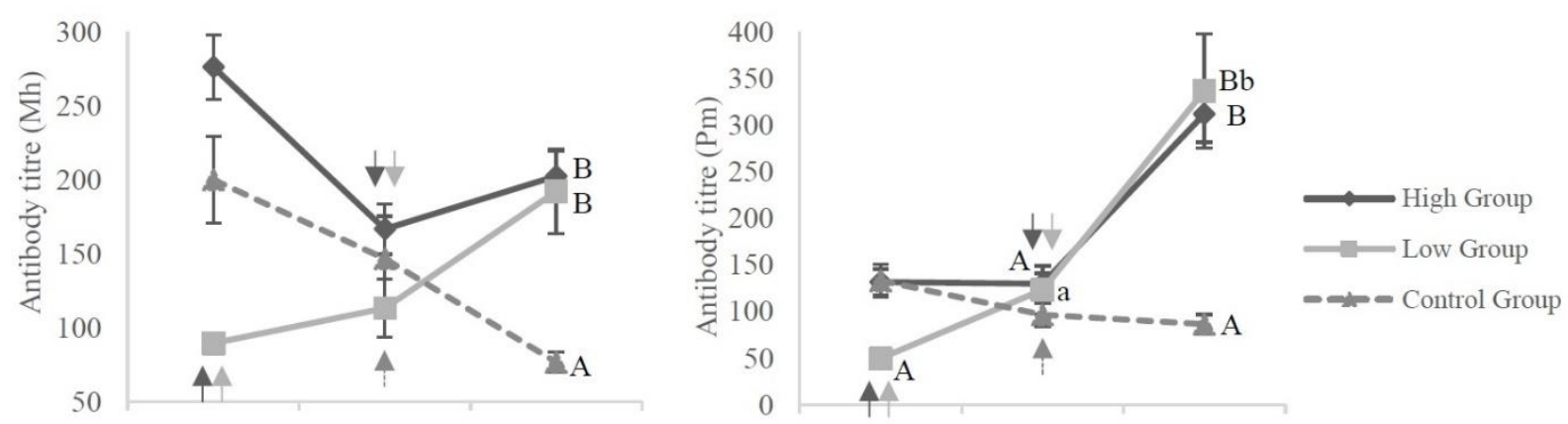

(2)
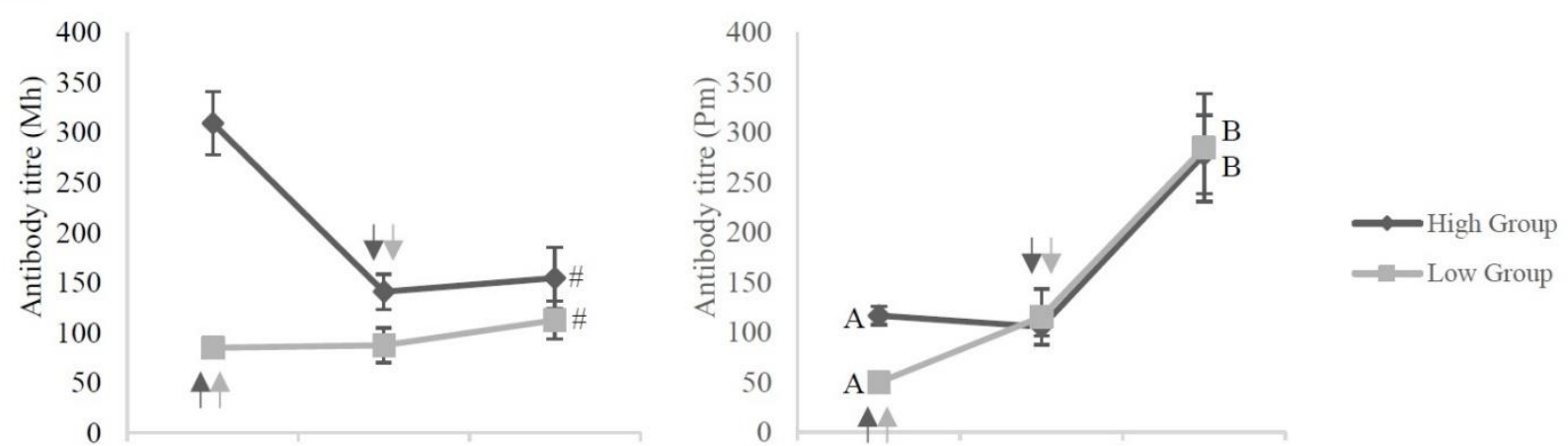

Fig. 4. Antibody response to the vaccination among calves with different maternal antibody levels (MAL)

Antibody titres against $M$. haemolytica $(\mathrm{Mh})$ and $P$. multocida $(\mathrm{Pm})$ in (1) the first experiment and in (2) the second experiment for the high, low, and control groups. A, B P $<0.01$; $\mathrm{a}, \mathrm{b} \mathrm{P}<0.05$; \# $\mathrm{P}=0.18$; arrows - time of vaccination

However, calves in the 5-6 day group did not show increased antibody production against $M$. haemolytica. The percentage of $M$. haemolytica-positive calves in the 7-12 day group was significantly higher than that in the 5-6 day group. No differences were found in the percentages of $H$. somni- or $P$. multocida positive calves between the 7-12 day group and the 5-6 day group (Table 2).

The titre against $M$. haemolytica at three weeks after the booster vaccination in the M. haemolytica high group was higher than that in the low group in the second experiment $(\mathrm{P}=0.18)$ and Figure 4 represents this finding. There were no differences in the titres against $M$. haemolytica among groups at this time point in the first experiment. There were also no differences found in the titres against $P$. multocida at three weeks after the booster dose in either experiment (Fig. 4). The percentages of $M$. haemolytica-positive calves in the high group were higher than those in the low group in the first $(\mathrm{P}<0.05)$ and second experiments $(\mathrm{P}=0.14)$. No differences were indicated between the high and low groups in the percentages of $P$. multocida-positive calves at three weeks after the booster dose in either experiment (Table 2).

\section{Discussion}

This research showed that antibody titres increased in the young calves after the booster dose of the inactivated bacterial vaccine. Although the titre against $M$. haemolytica did not significantly increase, the titre 
after the booster dose was significantly higher than that in the control group. Kato et al. reported that calves with a single injection of inactivated $M$. haemolytica vaccine at five months of age exhibited increased antibody titres. There was no significant difference in antibody titres, when determined within one month of the last injection, between two groups of Holstein dairy calves having in one case a single injection at five months of age and in the other one injection at one month and another at five months of age (11). In contrast, another study concluded that an inactivated vaccine needs multiple doses in order to obtain sufficient responses (2). Furthermore, several reports showed that BRD pathogenic bacteria such as H. somni, P. multocida, and M. haemolytica are part of the normal intranasal flora $(1,12)$. These pathogens usually induce significant disease if other predisposing environmental factors, physiologic stressors, or concurrent infections are present $(6,8)$. Based on the results of Kato et al. (11), it can be surmised that fivemonth-old calves might have received chronic antigenic stimulation by those bacteria, which could have acted as a replacement for the primary vaccination. In summary, administering an inactivated bacterial vaccine to young Holstein calves at one month of age or younger could produce antibodies with a booster dose.

Moreover, calves with high (the M. haemolytica high group) and low MAL (the M. haemolytica low group) showed similar antibody titres against this bacterium at three weeks after the booster dose. In addition, the percentage of $M$. haemolytica-positive calves at three weeks after the booster dose in the high group exceeded that in the low group. Çokçalışkan et al. showed that an inactivated foot-and-mouth disease vaccine, preferably followed by a booster vaccination, could overcome maternal immunity (4). We concluded that vaccination of younger calves with an inactivated bacterial vaccine could overcome transferred maternal immunity when administered with a booster dose.

We also found that the majority of M. haemolyticapositive calves in the first experiment were vaccinated at seven days of age or older. Surlis et al. reported that in young Holstein calves, chemokine expression is upregulated at the age of seven days compared to its expression level at two or three days Top upstream regulators of chemokine production include granulocyte-macrophage colony-stimulating factor (GM-CSF) which encodes granulocyte stimulating factor, a cytokine that stimulates the differentiation of haematopoietic precursor cells such as granulocytes and macrophages (19). Additionally, Kennedy et al. showed that serum GM-CSF in humans at three days after receiving an inactivated influenza vaccine showed correlation with 28-day post vaccination HAI and VNA titres which have been conventionally used to indicate immunologic response to vaccination in humans (13). Thus, we reasoned that calves might have a hindered ability to respond to a vaccine antigen at the age of five or six days. Although calves which received the primary vaccination at this time (the 5-6 day group) had higher antibody titres against $M$. haemolytica than the control group at three weeks after the booster dose, the titre against $M$. haemolytica did not show an increase as a result of the vaccinations of 5-6-day-old calves. In contrast, calves which received the primary dose at 7-12 days of age (the 7-12 day group) exhibited increased antibody titres against not only $H$. somni and P. multocida but also against $M$. haemolytica. Therefore, we propose that Holstein calves should receive the primary vaccination at seven days of age or later.

In conclusion, the booster dose of the inactivated $H$. somni, P. multocida, and M. haemolytica vaccine increased antibody production in young Holstein calves. The booster vaccination overcame maternal immunity, and calves should be vaccinated at seven days of age or older. However, $27.5 \%$ of calves were still seronegative at three weeks after the booster dose in the group which received the primary dose at the age of 7-12 days (the 7-12 day group). This high negative percentage did not fall with the delay of the primary vaccination by one or two weeks (in the second experiment). The conditions required in order for young Holstein calves to produce antibodies against $M$. haemolytica were not clarified. Further studies are needed in order to elucidate how best to protect young calves from BRD.

Conflict of Interests Statement: The authors declare that there is no conflict of interests regarding the publication of this article.

Financial Disclosure Statement: The vaccines were provided by and antibody levels were measured by Kyoto Biken Laboratories, Inc. at no charge.

Animal Rights Statement: All animals were regular patients of the Farm Animal Clinical Services located at the Rakuno Gakuen University Animal Medical Center. All procedures and animal care provided in the study were in accordance with the guidelines approved by the Rakuno Gakuen University Animal Experiment and Care Committee.

Acknowledgements: We thank Dr. Shuichi Kubota (Kyoto Biken Laboratories, Inc.) for his support in this study. Our appreciation also goes to the farm owners and all personnel of the enrolled farm for their cooperation.

\section{References}

1. Allen J.W., Viel L., Bateman K.G., Rosendal S., Shewen P.E., Physick-Sheard P.: The microbial flora of the respiratory tract in feedlot calves: associations between nasopharyngeal and bronchoalveolar lavage cultures. Can J Vet Res 1991, 55, 341-346.

2. Baxter D.: Active and passive immunity, vaccine types, excipients and licensing. Occup Med 2007, 57, 552-556.

3. Brumbaugh G.W.: Will antimicrobial resistance of BRD pathogens impact BRD management in the future? Anim Health Res Rev 2014, 15, 175-177. 
4. Çokçalıșkan C., Türkoğlu T., Uzunlu E., Sareyyüpoğlu B., Hancı İ., İpek A., Arslan A., Babak A., İldeniz G., Gülyaz V.: Influence of vaccine potency and booster administration of footand-mouth disease vaccines on the antibody response in calves with maternal antibodies. J Vet Sci 2017, 18, 315-322.

5. Confer A.W.: Update on bacterial pathogenesis in BRD. Anim Health Res Rev 2009, 10, 145-148

6. Earley B., Buckham Sporer K., Gupta S.: Invited review: Relationship between cattle transport, immunity, and respiratory disease. Animal 2017,11, 486-492.

7. Foote M.R., Nonnecke B.J., Beitz D.C., Waters W.R.: Antigenspecific B-cell responses by neonatal calves after early vaccination. J Dairy Sci 2007, 90, 5208-5217.

8. Griffin D., Chengappa M.M., Kuszak J., McVey D.S.: Bacterial pathogens of the bovine respiratory disease complex. Vet Clin North Am Food Anim Pract 2010, 26, 381-394.

9. Hodgins D.C., Shewen P.E.: Serologic responses of young colostrum fed dairy calves to antigens of Pasteurella haemolytica A1. Vaccine 1998, 16, 2018-2025.

10. Kampen A.H., Olsen I., Tollersrud T., Storset A.K., Lund A.: Lymphocyte subpopulations and neutrophil function in calves during the first 6 months of life. Vet Immunol Immunopathol 2006, 113, 53-63.

11. Kato H., Sugiyama M., Sakuma M., Yoneyama O., Sato R., Oonishi M., Tajima M.: Antibody response to Mannheimia haemolytica vaccination in young Holstein calves. J Vet Med 2012, 65, 694-697.

12. Kato T., Yamamoto T., Ogata Y., Urushiyama Y., Ogino S., Saito H.: Clinical effectiveness of programs for treatment of respiratory diseases in cattle based on drug susceptibility. J Jpn Vet Med Assoc 2008, 61, 294-298

13. Kennedy R.B., Tosh P.K., Goergen K.M., Grill D.E., Oberg A.L., Poland G.A.: Statistical modeling using early markers of innate immunity to explain variation in humoral responses to influenza vaccine in older adults. Vaccine 2015, 33, 3682-3688.

14. Kramer L.M., Mayes M.S., Fritz-Waters E., Williams J.L., Downey E.D., Tait R.G., Woolums A., Chase C., Reecy J.M.:
Evaluation of responses to vaccination of Angus cattle for four viruses that contribute to bovine respiratory disease complex. J Anim Sci 2017, 95, 4820-4834.

15. Nagai K., Otomaru K., Ogawa R., Oishi S., Wataya K., Honkawa Y., Iwamoto Y., Ando T., Hyakutake K., Shirahama H., Habiby G., Kubota C.: Effect of combined vaccination for Pasteurella multocida, Mannheimia haemolytica, and Histophilus somni to prevent respiratory diseases in young Japanese Black calves in the field. J Vet Med Sci 2019, doi: 10.1292/jvms.19-0256.

16. Nonnecke B.J., Waters W.R., Foote M.R., Palmer M.V., Miller B.L., Johnson T.E., Perry H.B., Fowler M.A.: Development of an adult-like cell-mediated immune response in calves after early vaccination with Mycobacterium bovis bacillus CalmetteGuérin. J Dairy Sci 2005, 88, 195-210.

17. Otomaru K., Kubota S., Ohtsuka H., Ando T., Koiwa M.: Field application of mixed vaccine including inactivated Pasteurella multocida, Mannheimia haemolytica and Histophilus somni to Japanese Black calves for preventing development of respiratory disease. J Jpn Vet Med Assoc 2012, 65, 767-770.

18. 18. Otomaru K., Ohtsuka H., Ando T., Koiwa M.: Evaluation of the effect of Mannheimia haemolytica inactivation vaccine on Japanese Black cattle in the field. J Jpn Vet Med Assoc 2011, 64, 787-790

19. Surlis C., Earley B., McGee M., Keogh K., Cormican P., Blackshields G., Tiernan K., Dunn A., Morrison S., Arguello A., Waters S.M.: Blood immune transcriptome analysis of artificially fed dairy calves and naturally suckled beef calves from birth to 7 days of age. Sci Rep 2018, 8, 15461.

20. USDA-APHIS-VS-CEAH-NAHMS.: Health and management practices on U.S. Dairy Operations, 2014. U.S.D.A., Fort Collins, 2018.

21. Waltner-Toews D., Martin S.W., Meek A.H.: The effect of early calfhood health status on survivorship and age at first calving. Can J Vet Res 1986, 50, 314-317. 